\title{
Markov properties of cluster processes
}

\author{
A J Baddeley ${ }^{1,2}$ \\ M N M van Lieshout ${ }^{3,4}$ \\ J Møller ${ }^{5}$ \\ 1 Department of Mathematics, University of Western Australia, Nedlands WA 6009, Australia \\ 2 Department of Mathematics and Computer Science, University of Leiden, The Netherlands \\ 3 Department of Mathematics and Computer Science. Free University \\ De Boelelaan 1081 a, 1081 HV Amsterdam. The Netherlands \\ $4 \mathrm{CWI}$, Amsterdam, The Netherlands \\ ${ }^{5}$ Department of Theoretical Statistics, University of Aarhus, Ny Munkegade, DK-8000 Aarhus C, Denmark
}

\section{Abstract}

We show that a Poisson cluster point process is a nearest-neighbour Markov point process [2] if the clusters have uniformly bounded diameter. It is typically not a finite-range Markov point process in the sense of Ripley and Kelly [11]. Furthermore, when the parent Poisson process is replaced by a Markov or nearest-neighbour Markov point process, the resulting cluster process is also nearest-neighbour Markov, provided all clusters are nonempty. In particular, the nearest-neighbour Markov property is preserved when points of the process are independently randomly translated, but not when they are randomly thinned.

\section{INTRODUCTION}

Markov or Gibbs point processes $[2,8,11,12]$ form a large, flexible, and understandable class of point process models with many practical advantages (see e.g. $[4,9,10]$ for surveys). In this paper we consider the relationship of these models to the basic point process operation of clustering. We ask whether cluster processes are Markov, and whether the Markov property is preserved under clustering.

In a Poisson cluster process, intuitively the only 'spatial dependence' present is that between offspring of the same parent. If the offspring of a given parent all lie within distance $R$ of the parent, then two offspring of the same parent lie at most $2 R$ apart, and it is plausible to conjecture that the process is Markov with finite interaction range $2 R$ in the sense of Ripley and Kelly [11].

However, this turns out to be false in general, because certain spatial configurations of the offspring points imply information about the unobserved parent points, and this information can 'propagate' over arbitrarily large distances.

In this paper we show that cluster processes have the nearest-neighbour Markov property in the sense of Baddeley \& Møller [2]. We prove that (a) any Poisson cluster process with uniformly bounded clusters is a nearest-neighbour Markov point process; and (b) if a Markov or nearest-neighbour Markov point process is used as the parent process for a cluster process, 
and the clusters are uniformly bounded and a.s. nonempty, then the cluster process is again nearest-neighbour Markov. In particular, the nearest-neighbour Markov property is preserved under random displacement of points, but not under random thinning.

These results support the claim [6,7] that nearest-neighbour Markov processes (as opposed to Ripley-Kelly Markov processes) provide a rich class of models for clustering, and further suggest that they may include good models for multiple-generation cluster processes, $\mathrm{cf}$. [5]. Result (a) may also explain why statistical theory for Poisson cluster processes so closely parallels that for Markov point processes [1].

The next section recalls standard definitions; the main results are stated in Section 3 and the proofs follow in Section 4.

\section{SETUP}

\subsection{Point processes}

We consider finite point processes $X$ on a metric space $S$ (typically $\mathbb{R}^{d}$ or a compact subset). Each realization of such a process "is" a finite set $\mathbf{x}=\left\{x_{1}, \ldots, x_{n}\right\}$ of points $x_{i} \in S$ with $n \geq 0$. Strictly speaking the points may be multiply occupied, and $n$ is the total multiplicity, but this will have probability zero in the applications considered. Realizations will also be called 'configurations' and the class of all configurations will be denoted by $\mathcal{C}$. This is the exponential space of $S$, see [3] or [2] for details.

Let $\nu$ be some given Borel measure on $S$ (typically Lebesgue measure); we will consider processes whose distributions are absolutely continuous with respect to the measure $\mu$ on $\mathcal{C}$ defined by

$$
\mu(F)=\sum_{k=0}^{\infty} \frac{1}{k !} \int \ldots \int 1\left[\left\{y_{1}, \ldots, y_{k}\right\} \in F\right] d \nu\left(y_{1}\right) \ldots d \nu\left(y_{k}\right)
$$

If $\nu$ is totally finite (e.g. if $S$ is compact and $\nu$ is Lebesgue measure), then $\mu$ is $e^{\nu(S)}$ times the distribution of the Poisson process on $S$ with intensity measure $\nu$.

Let $f: \mathcal{C} \rightarrow[0, \infty)$ be the density of a point process $X$ with respect to $\mu$. We say $f$ is hereditary if

$$
f(\mathbf{x})>0 \text { implies } f(\mathbf{z})>0 \text { for all } \mathbf{z} \subset \mathbf{x}
$$

and hereditary excluding $\emptyset$ if this holds except when $\mathbf{z}=\emptyset$.

2.2 Markov point processes

This subsection collects necessary definitions from $[2,9,11]$.

Define $u, v \in S$ to be $r$-close, written $u \sim v$, if $d(u, v)<r$ where $d$ is the metric of $S$. This defines a relation $\sim$ on $S$ which is clearly symmetric and reflexive. [The results of this paper extend to the case where $\sim$ is any symmetric reflexive relation on $S$ which is measurable in the product space. Theorem 2 in Section 3 requires two such relations. ]

Definition 1 (Ripley-Kelly) A point process $\mathrm{X}$ is Markov with respect to the static relation $\sim$ if its density $f$ satisfies

- $f$ is hereditary; 
- $f(u,: n ! \mathrm{x} \in \mathcal{C}$ such that $f(\mathrm{x})>0$ and $u \in S$, the ratio

$$
\frac{f(\mathbf{x} \cup\{u\})}{f(\mathbf{x})}
$$

depends only on $u$ and on $\left\{x_{i} \in \mathrm{x}: u \sim x_{i}\right\}$.

Now define for each $x \in \mathcal{C}$ the "connected component relation" [2, Appendix III] between points of $\mathbf{x}$ by

$$
x_{i} \underset{\mathrm{x}}{\sim} x_{j} \quad \text { iff } \quad x_{i} \sim z_{1} \sim \cdots \sim z_{n} \sim x_{j} \text { for some } z_{1}, \ldots, z_{n} \in \mathbf{x}
$$

In other words, two points of $x$ are related under $\tilde{x}$ if they are in the same connected c'mponent of the finite graph whose edges connect every pair of $r$-close points in $\mathbf{x}$.

Definition 2 (Baddeley-Møller) A point process $X$ is nearest-neighbour Markov with respect to the dynamic relation $\tilde{\mathrm{x}}$ if its density $f$ is hereditary and the ratio (2.2) depends only on $u$, on

$$
\operatorname{Nbd}(u \mid \mathbf{x} \cup\{u\})=\left\{x_{i} \in \mathbf{x}: u \tilde{\mathbf{x}} x_{i}\right\},
$$

and on the relations $\tilde{\mathbf{x}}, \quad \mathbf{x} \cup\{\mathbf{u}\}$ restricted to $\operatorname{Nbd}(u \mid \mathbf{x} \cup\{u\})$.

Clearly if $X$ is Markov with respect to $\sim$, it is also nearest-neighbour Markov with respect to both $\sim$ and $\tilde{x}$.

Analogues of the Hammersley-Clifford theorem proved in [11] and in [2] give explicit expressions for the density $f$ when $X$ is Markov and nearest-neighbour Markov, respectively. Define a configuration $\mathbf{z}$ to be a clique with respect to $\sim$ (or an $r$-clique) if all pairs of points in $\mathbf{z}$ are $r$-close, $z_{i} \sim z_{j}$ for all $z_{i}, z_{j} \in \mathbf{z}$. Then [11] $X$ is Markov iff

$$
f(\mathbf{x})=\prod_{\mathbf{z} \subseteq \mathbf{x}} \varphi(\mathbf{z})
$$

where $\varphi(\mathbf{z}) \geq 0$ with $\varphi(\mathbf{z}) \neq 1$ only if $\mathbf{z}$ is a clique.

In the nearest-neighbour case, a subconfiguration $\mathbf{z} \subseteq \mathbf{x}$ is termed a clique with respect to $\tilde{\mathrm{x}}$ if all pairs of points in $\mathbf{z}$ are $\tilde{\mathrm{x}}$-neighbours, $z_{i} \tilde{\mathrm{x}} z_{j}$ for all $z_{i}, z_{j} \in \mathbf{z}$. The maximal $r$-cliques are also called connected components.

An analogue of (2.3) for dynamic relations $\tilde{x}$ is given in [2, Theorem 4.13]. For the 'connected component relation' this specialises to the following result.

Lemma 1 A point process $X$ is nearest-neighbour Markov with respect to the connected component relation $\tilde{\mathrm{x}}$ iff

$$
f(\mathbf{x})=\prod_{\text {cliques } \mathbf{z} \subseteq \mathbf{x}} \varphi(\mathbf{z})
$$

where $\varphi(\cdot) \geq 0$ is such that whenever $\mathbf{z}$ is a $\underset{\mathbf{z}}{\sim}$-clique with $\varphi(\mathbf{z})>0$ then $\varphi(\mathbf{w})>0$ for all $\mathbf{w} \subset \mathbf{z}$. 
For example, for a Matérn cluster process, in the case $s \leq 2 R$ consider a configuration of three points $y_{1}, y_{2}, y_{3}$ such that $\left\|y_{1}-y_{2}\right\|<s,\left\|y_{2}-y_{3}\right\|<s$, but $\left\|y_{1}-y_{3}\right\|>s$. If $f$ were a Markov function at range $s$ then

$$
f\left(\left\{y_{1}, y_{2}, y_{3}\right\}\right) f\left(\left\{y_{2}\right\}\right)=f\left(\left\{y_{1}, y_{2}\right\}\right) f\left(\left\{y_{2}, y_{3}\right\}\right) .
$$

Substituting (3.7) gives

$$
\left[1+e^{\omega} J\left(y_{1}, y_{2}\right)+e^{\omega} J\left(y_{2}, y_{3}\right)\right]=\left[1+e^{\omega} J\left(y_{1}, y_{2}\right)\right]\left[1+e^{\omega} J\left(y_{2}, y_{3}\right)\right]
$$

This is clearly a contradiction, since the $J$ terms are nonzero. Hence $f$ is not a Markov density in the Ripley-Kelly sense at distance $s$. For $s>2 R$ one can use similar arguments involving chains of more than three points.

Next we consider cluster processes generated from a parent process which is Markov or nearest-neighbour Markov. In general the cluster process is not Markov.

Counterexample 2 Let $\mathbf{x}$ be a Ripley-Kelly Markov point process (finite range $r$ ) and $\mathbf{y}$ the result of thinning the points independently with retention probability $q, 0<q<1$. Then, in general, $\mathrm{y}$ is not a nearest-neighbour Markov point process (and a fortiori it is not a Ripley-Kelly Markov process) for any $R<\infty$.

This can be checked from (2.6), since random thinning is the special case of clustering in which $Z_{\xi}=\{\xi\}$ with probability $q$, and $Z_{\xi}=\emptyset$ otherwise. For any given pair of points $y_{i}, y_{j} \in \mathbf{y}$ there are (potentially) nonzero summands in (2.6) of the form $q_{x_{1}}\left(y_{i}\right) q_{x_{2}}(\emptyset) \cdots q_{x_{N-1}}(\emptyset) q_{x_{N}}\left(y_{j}\right)$ involving both $y_{i}, y_{j}$. Hence $\mathbf{y}$ is not nearest-neighbour Markov according to (2.4).

Clearly this problem may arise whenever clusters are permitted to be empty, i.e. when a parent point may have no offspring. When this is excluded, we do obtain a Markov property.

Theorem 2 Let $\mathbf{x}$ be a Markov or nearest-neighbour Markov point process at range $r$ and $\mathbf{y}$ the associated cluster process satisfying $(A)-(D)$ of section 2.3 and moreover

(E) the clusters are nonempty a.s.

Then $\mathrm{y}$ is a nearest-neighbour Markov point process for the connected component relation at range $2 R+r$.

Corollary 1 Let $\mathbf{x}$ be as above, and let $\mathbf{y}$ be the process obtained from $\mathbf{x}$ by independently translating each point: $y_{i}=x_{i}+v_{i}$, where the vectors $v_{i}$ are i.i.d., have a probability density, and satisfy $\left\|v_{i}\right\|<R$ a.s. Then $\mathbf{y}$ is a neurest-neighbour Markov point process for the connected component relation at range $2 R+r$. 
4. ProOFs

Proof of Lemma 1:

Suppose that (2.4) holds. i.et $\mathbf{x} \in \mathcal{C}, \xi \in S$ and let $\mathbf{x}_{D_{1}}, \ldots, \mathbf{x}_{D_{K}}$ and $\mathbf{w} \cup\{\xi\}$ denote the connected components of $\mathbf{x} \cup\{\xi\}$. Then, if $\mathbf{x}_{D_{K+1}}, \ldots, \mathbf{x}_{D_{L}}$ are the connected components of $\mathbf{w}$, we have that $\mathbf{x}_{D_{1}}, \ldots, \mathbf{x}_{D_{L}}$ are the connected components of $\mathbf{x}$, and

while

$$
f(\mathbf{x} \cup\{\xi\})=\varphi(\emptyset)\left[\prod_{i=1}^{K} \prod_{\emptyset \neq \mathbf{z} \subseteq \mathbf{x}_{D_{i}}} \varphi(\mathbf{z})\right] \prod_{\emptyset \neq \mathbf{z} \subseteq \mathbf{w} \cup\{\xi\}} \varphi(\mathbf{z})
$$

$$
f(\mathbf{x})=\varphi(\emptyset)\left[\prod_{i=1}^{K} \prod_{\emptyset \neq \mathbf{z} \subseteq \mathbf{x}_{D_{\mathbf{s}}}} \varphi(\mathbf{z})\right] \prod_{j=K+1}^{L} \prod_{\emptyset \neq \mathbf{z} \subseteq \mathbf{x}_{D_{j}}} \varphi(\mathbf{z}) .
$$

Hence $f(\mathbf{x} \cup\{\xi\})>0$ implies $f(\mathbf{x})>0$ (as $\mathbf{z} \subseteq \mathbf{x}_{D_{j}}$ for $j>K$ implies that $\mathbf{z} \subseteq \mathbf{w}$ ) and $f(\mathbf{x} \cup\{\xi\}) / f(\mathbf{x})$ satisfies the conditions of Definition 2. Thus $X$ is nearest-neighbour Markov.

Conversely, suppose $X$ is nearest-neighbour Markov. By the analogue of the HammersleyClifford theorem [2, Theorem 4.13],

$$
f(\mathbf{x})=\prod_{\mathbf{y} \subseteq \mathbf{x}} \varphi(\mathbf{y})^{\chi(\mathbf{y} \mid \mathbf{x})} \quad\left(\text { taking } 0^{0}=0\right)
$$

where $\chi(\mathbf{y} \mid \mathbf{x})=1$ if $\mathbf{y}$ is a $\underset{\mathbf{x}}{\sim}$-clique and 0 otherwise; and $\varphi: \mathcal{C} \rightarrow \mathbb{R}_{+}$satisfies

(I1) $\varphi(\mathbf{x})>0$ implies $\varphi(\mathbf{y})>0$ for all $\mathbf{y} \subset \mathbf{x}$

(I2) $\varphi(\mathbf{x})>0$ and $\varphi(\{\xi\} \cup \operatorname{Nbd}(\xi \mid \mathbf{x} \cup\{\xi\}))>0$ imply $\varphi(\mathbf{x} \cup\{\xi\})>0$.

Note that, in the case of the connected component relation, $\xi \widetilde{y} \eta$ implies $\xi \widetilde{x} \eta$ for $\mathbf{x} \supseteq \mathbf{y}$, so that $\chi(\mathbf{y} \mid \mathbf{y})=1$ implies $\chi(\mathbf{y} \mid \mathbf{x})$ for any $\mathbf{x} \supseteq \mathbf{y}$.

To prove that (4.9) reduces to (2.4) we need to show that, if $\varphi(\mathbf{y})>0$ for all $\mathbf{y} \subseteq \mathbf{x}$ with $\chi(\mathbf{y} \mid \mathbf{x})=1$, then $\varphi(\mathbf{y})>0$ for all $\mathbf{y} \subseteq \mathbf{x}$.

To prove this, suppose $\mathbf{v}, \mathbf{w} \subset \mathbf{x}$ are disjoint connected components of $\mathbf{x}$ (i.e. with respect to $\sim$ ). If $\xi \in \mathbf{v}$ then $\operatorname{Nbd}(\xi \mid \mathbf{w} \cup\{\xi\})=\{\xi\}$, and by assumption $\varphi(\{\xi\})>0$, so (I2) gives $\varphi(\mathbf{w} \cup\{\xi\})>0$. Similarly if $\{\xi, \eta\} \subseteq \mathbf{v}$ then $\operatorname{Nbd}(\eta \mid \mathbf{w} \cup\{\xi, \eta\})=\{\xi, \eta\}$, and by assumption $\varphi(\{\xi, \eta\})>0$, so (I2) gives $\varphi(\mathbf{w} \cup\{\xi, \eta\})>0$. Continuing in this way we obtain that $\varphi(\mathbf{y})>0$ for all $\mathbf{y} \subseteq \mathbf{x}$.

Hence if $X$ is nearest-neighbour Markov then its density is of the form (2.4) where $\varphi$ satisfies (I1) and hence the condition stated in the Lemma.

$\square$

\section{Proof of Lemma 2:}

The clusters $Z_{i}$ being conditionally independent given $\mathbf{x}$, we have for any measurable event F

$$
\begin{aligned}
\mathbb{P}\{\mathbf{y} \in F \mid \mathbf{x}\} & =\int \mathbf{1}\left[\bigcup_{i} Z_{i} \in F\right] q_{x_{1}}\left(Z_{1}\right) \cdots q_{x_{n}}\left(Z_{n}\right) d \mu\left(Z_{1}\right) \ldots d \mu\left(Z_{n}\right) \\
& =\sum_{C_{1}, \ldots, C_{n}} \int \mathbf{1}[\mathbf{y} \in F] q_{x_{1}}\left(\mathbf{y}_{C_{1}}\right) \cdots q_{x_{n}}\left(\mathbf{y}_{C_{n}}\right) d \mu(\mathbf{y}) .
\end{aligned}
$$


The last line was obtained using (2.1) by rewriting each integral over $Z_{i}$ as a sum of multiple integrals with respect to $\nu$ and regrouping. The result follows by taking expectations with respect to $\mathbf{x}$.

\section{Proof of Theorem 1:}

By (2.6), the density of $\mathbf{y}$ with respect to $\mu$ is (for $\mathbf{y} \neq \emptyset$ )

$$
\begin{aligned}
f(\mathbf{y}) & =\sum_{n=1}^{\infty} \frac{e^{-\lambda(S)}}{n !} \int_{S} \ldots \int_{S} \sum_{C_{1}, \ldots, C_{n}} \prod_{i=1}^{n} q_{x_{i}}\left(\mathbf{y}_{C_{i}}\right) d \lambda\left(x_{1}\right) \cdots d \lambda\left(x_{n}\right) \\
& =\sum_{n=1}^{\infty} \frac{e^{-\lambda(S)}}{n !} \sum_{C_{1}, \ldots, C_{n}} \prod_{i=1}^{n} \int_{S} q_{\xi}\left(\mathbf{y}_{C_{i}}\right) d \lambda(\xi)
\end{aligned}
$$

here the inner sum is over all ordered partitions of $\mathbf{y}$ into $n$ disjoint, possibly empty, sets. Since the parent process is Poisson, the number of non-empty clusters is Poisson distributed with mean $\beta=\int_{S}\left(1-q_{\xi}(\emptyset)\right) d \lambda(\xi)$, so that for $\mathbf{y}=\emptyset$ we have $f(\emptyset)=e^{-\beta}$.

Now $q_{\xi}(\mathbf{z})=0$ whenever $\mathbf{z} \not \subset b(\xi, R)$; hence if $q_{\xi}(\mathbf{z}) \neq 0$ then all pairs of points in $\mathbf{z}$ are. $2 R$-close, i.e. $\mathbf{z}$ is a clique with respect to the finite range relation with distance $2 R$. Hence the integral in (4.11) is nonzero only when the partition consists of $2 R$-cliques.

For $\mathbf{y} \neq \emptyset$, let $\mathbf{y}_{D_{1}}, \ldots, \mathbf{y}_{D_{K}}$ be the connected components of $\mathbf{y}$ for the relation $\sim$ with range $2 R$. Then the integral in (4.11) is nonzero only when the partition is a refinement of $D_{1}, \ldots, D_{K}$. Let $C_{1}, \ldots, C_{k}$ be an (unordered) partition refining $D_{1}, \ldots, D_{K}$ and consisting of non-empty sets. This contributes a term

$$
\alpha \prod_{i=1}^{k} \int_{S} q_{\xi}\left(\mathbf{y}_{C_{i}}\right) d \lambda(\xi)
$$

to the density. Since $\int_{S} q_{\xi}(\emptyset) d \lambda(\xi)=\lambda(S)-\beta$, the coefficient $\alpha$ is

$$
\sum_{n=k}^{\infty} \frac{e^{-\lambda(S)}}{n !}(\lambda(S)-\beta)^{n-k} n(n-1) \cdots(n-k+1)=e^{-\beta}
$$

The class of all partitions that are refinements of $D_{1}, \ldots, D_{K}$ is the Cartesian product of the sets of partitions of each $D_{i}$. Hence, for $\mathbf{y} \neq \emptyset$,

$$
f(\mathbf{y})=e^{-\beta} \prod_{i=1}^{K} \Phi\left(\mathbf{y}_{D_{i}}\right)
$$

where

$$
\Phi(\mathbf{z})=\sum_{k \geq 1} \sum_{\mathbf{z}_{C_{1}}, \ldots, \mathbf{z}_{C_{k}}} \prod_{j=1}^{k} \int_{S} q_{\xi}\left(\mathbf{z}_{C_{j}}\right) d \lambda(\xi)
$$

where $\mathbf{z}_{C_{1}}, \ldots, \mathbf{z}_{C_{k}}$ range over all (unordered) partitions of $\mathbf{z}$ into nonempty subconfigurations. Since the offspring densities $q_{\xi}$ are hereditary excluding $\emptyset$, clearly $\Phi$ is hereditary excluding $\emptyset$, and hence $f$ is hereditary. According to (2.4) the density (4.12) is nearest-neighbour Markov with respect to the connected component relation at range $2 R$. $\square$ 
Proof of Theorem 2: The density $p(\mathbf{x})$ of $\mathbf{x}$ can be factorised as in (2.4). By (2.6), the density of $y$ with respect to $\mu$ is

$$
f(\mathbf{y})=\int_{\mathcal{C}^{\prime}} \sum_{\mathcal{C}_{1} \ldots, C_{n}(\mathbf{x})} \prod_{i=1}^{n(\mathbf{x})} q_{x_{1}}\left(\mathbf{y}_{C_{2}}\right) p(\mathbf{x}) d \mu(\mathbf{x})
$$

where the sum ranges over all ordered partitions of $y$ into disjoint, possibly empty subsets. Since $q_{\xi}(())=0$. the int egrand of $(4.14)$ can be rephrased as

$$
\sum_{i} \prod_{i=1}^{n} q_{x_{i}}\left(\mathbf{y}_{t^{-1}(i)}\right) p(\mathbf{x})
$$

Where 6 ranges over all surjective mappings of the points of $\mathbf{y}$ onto the points of $\mathbf{x}$, identified with mappings from $\{1, \ldots . m\}$ onto $\{1, \ldots, n\}$.

Wo can restrict attention to those $\epsilon$ such that

$$
d\left(y_{i}, x_{\epsilon(i)}\right) \leq R \quad \text { for all } i
$$

since all other terms are zero. For such $\epsilon$, if $\mathbf{z} \subseteq \mathbf{x}$ is an $r$-clique and $\epsilon^{-1}(\mathbf{z})=\mathbf{w} \subseteq \mathbf{y}$, then w must be a $(2 R+r)$-clique. To see this, take $y_{i}, y_{j} \in \mathbf{w}$ and apply the triangle inequality:

$$
\begin{aligned}
d\left(y_{i}, y_{j}\right) & \leq d\left(y_{i}, x_{\epsilon(i)}\right)+d\left(x_{\epsilon(i)}, x_{\epsilon(j)}\right)+d\left(x_{\epsilon(j)}, y_{j}\right) \\
& \leq R+r+R .
\end{aligned}
$$

By a similar arg:ment, if $\mathbf{z} \subseteq \mathbf{x}$ is a clique with respect to the relation $\sim$ at distance $r$, then $\mathrm{w}$ is a clique with respect to the relation $\tilde{y}_{\mathrm{y}}$ at distance $2 R+r$.

Let $\mathbf{y}_{D_{1}}, \ldots, \mathbf{y}_{D_{K}}$ be the connected components of $\mathbf{y}$ with respect to the relation at distance $2 R+r$. Then we can rewrite $(4.15)$ as

$$
\begin{aligned}
& \prod_{\epsilon} \rho(\mathbf{z}) \sum_{\epsilon} \prod_{i=1}^{n} q_{x_{i}}\left(\mathbf{y}_{\epsilon^{-1}(i)}\right) \\
= & \sum_{\epsilon}\left[\prod_{i=1}^{n} q_{x_{i}}\left(\mathbf{y}_{\epsilon^{-1}(i)}\right) \prod_{k=1}^{K} \operatorname{cliques} \prod_{\mathbf{z} \subseteq \mathbf{x}: \epsilon^{-1}(\mathbf{z}) \subseteq D_{k}} \varphi(\mathbf{z})\right] \\
= & \sum_{\epsilon} \prod_{k=1}^{K}\left[\prod_{i: \epsilon^{-1}(i) \subseteq D_{k}} q_{x_{i}}\left(\mathbf{y}_{\epsilon^{-1}(i)}\right) \prod_{\operatorname{cliques} \subseteq \mathbf{z}: \epsilon^{-1}(\mathbf{z}) \subseteq D_{k}} \varphi(\mathbf{z})\right]
\end{aligned}
$$

Any $\epsilon$ of the type described above can be represented as an ordered set of $K$ surjective mappings

$$
\epsilon_{k}: D_{k} \rightarrow D_{k}^{\prime}=\left\{i \mid d\left(x_{i}, y_{j}\right) \leq R \text { for some } j \in D_{i}\right\}
$$

automatically satisfying the norm condition (4.16). Note that $\mathbf{x}_{D_{k}^{\prime}}, k=1, \ldots, K$ form a disjoint partition of $\mathbf{x}$. Thus $(4.17)$ is 


$$
\prod_{k=1}^{K} \sum_{\epsilon_{k}}\left[\prod_{i \in D_{k}^{\prime}} q_{x_{i}}\left(\mathbf{y}_{\epsilon_{k}^{-1}(i)}\right) \prod_{\text {cliques } \mathbf{z} \subseteq \mathbf{x}_{D_{k}^{\prime}}} \varphi(\mathbf{z})\right] .
$$

Integrating over $\mathbf{x}$ and exploiting the form (2.1) of $\mu$ yields

$$
f(\mathbf{y})=\prod_{k=1}^{K} \int_{C} \sum_{\epsilon_{k}} \prod_{i=1}^{n(\mathbf{v})} q_{x_{i}}\left(\mathbf{y}_{\epsilon_{k}^{-1}(i)}\right) \prod_{\text {cliques } \mathbf{z} \subseteq \mathbf{v}} \varphi(\mathbf{z}) d \mu(\mathbf{v}) .
$$

Thus, $f$ factorises as required by (2.4). The hereditary property follows as in the previous proof.

$\square$

\section{REFERENCES}

1. A.J. Baddeley and M.N.M. van Lieshout. A nonparametric measure of spatial interaction in point patterns. Research Report WS 417, Vrije Universiteit Amsterdam, december 1993.

2. A.J. Baddeley and J. Møller. Nearest-neighbour Markov point processes and random sets. International Statistical Review, 57:89-121, 1989.

3. D.S. Carter and P.M. Prenter. Exponential spaces and counting processes. Zeitschrift für Wahrscheinlichkeitstheorie und verwandte Gebiete, 21:1-19, 1972.

4. P.J. Diggle, T. Fiksel, Y. Ogata, D. Stoyan, and M. Tanemura. On parameter estimation for pairwise interaction processes. International Statistical Review, 1994. to appear.

5. J.F.C. Kingman. Remarks on the spatial distribution of a reproducing population. Journal of Applied Probability, 14:577-583, 1977.

6. J. Møller. Discussion contribution. Journal of the Royal Statistical Society, series B, 54:692-693, 1992.

7. J. Møller. Markov chain Monte Carlo and spatial point processes. Manuscript in preparation, 1994.

8. C.J. Preston. Random fields. Springer Verlag, Berlin-Heidelberg-New York, 1976.

9. B.D. Ripley. Statistical inference for spatial processes. Cambridge University Press, 1988.

10. B.D. Ripley. Gibbsian interaction models. In D.A. Griffiths, editor, Spatial statistics: past, present and future, pages 1-19. Image, New York, 1989.

11. B.D. Ripley and F.P. Kelly. Markov point processes. Journal of the London Mathematical Society, 15:188-192, 1977.

12. D. Ruelle. Statistical mechanics. John Wiley and Sons, New York, 1969. 